\title{
Uma análise bibliográfica e histórica entre serviços e design de serviços
}

\section{A literature review and historical analysis between services and service design}

\author{
Danieli Tartas ${ }^{1}$ \\ Eugenio Andrés Díaz Merino \\ Giselle Schmidt Alves Díaz Merino ${ }^{3}$ \\ Fernando Antonio Forcellini ${ }^{4}$
}




\section{Resumo}

O setor de serviços representa atualmente a principal atividade econômica para grande parte dos países desenvolvidos. O Design de Serviços é utilizado por muitas empresas para organizar, planejar e desenvolver pessoas, estruturas, materiais e informações, de forma a oferecer para os consumidores a melhor experiência possível ao usar um serviço. No entanto, para entender como ocorreu essa inserção do Design de Serviços dentro dos processos de desenvolvimento de serviços, sugere-se uma leitura e análise do histórico de ambos os conceitos. Com uma revisão sistemática da literatura e busca dos principais acontecimentos ao longo dos anos dentro das áreas, percebeu-se que a necessidade de ofertar para os clientes a melhor experiência com grande impacto na utilização de um serviço aumentou consideravelmente nos últimos 20 anos, e continua em expansão até 0 momento, crescimento também observado em publicações científicas. Nesse contexto, observa-se que o Design de Serviços surgiu como uma opção para que as empresas conseguissem visualizar e melhorar as experiências dos usuários ao utilizarem um serviço, de acordo com o período histórico vivenciado.

Palavras-Chave: Design de Serviços. Desenvolvimento de Serviços. Análise Histórica. Gestão de Design.

\section{Abstract}

The service sector represents currently the main economic activity for many developed countries. Service design is an option used by companies to organize, plan and develop people, structures, materials and informations, in order to provide to costumers the best experience using a service. However to understand how this integration between the Service Design and the service development activities happened it is suggested a reading and analysis through the history of both concepts. Through a systematic literature review and search by the main events over the years, it can be seen that the need to offer to customers the best experience with great impact on the use of a service increased considerably in the last 20 years and continues to expand to date, growth is also seen in scientific publications. In this context it can be understood that the Service Design emerged as an option for companies to visualize and improve experiences of costumers using a service, according to the historical period experienced.

Keywords: Service Design. Service Development. Historical Analysis. Design Management.

ISSN: 1808-3129

\footnotetext{
1 Programa de Pós Graduação em Engenharia de Produção - PPGEP Universidade Federal de Santa Catarina - UFSC, Brasil danielitartas@gmail.com 2 PPGEP/POSDESIGN - UFSC, Brasil eugenio.merino@ufsc.br

3 POSDESIGN - UFSC, Brasil gisellemerino@gmail.com

4 PPGEP - UFSC, Brasil forcellini@gmail.com
} 


\section{Introdução}

Segundo o IBGE (Instituto de Brasileiro de Geografia e Estatística) (2015), o setor de serviços representou em 2014 quase 70\% do PIB (Produto Interno Bruto) brasileiro, e é considerado o maior empregador do país. Apesar do crescimento deste setor, algumas vezes os processos de desenvolvimento de novos serviços não são adequadamente aplicados e resultam em falência.

O Design de Serviços pode ser visto como uma opção para que as empresas entendam melhor o cliente, a organização e o mercado, e com isso desenvolver soluções para oferecer a melhor experiência para os usuários em determinado serviço (MORITZ, 2005).

A análise histórica pode ser utilizada como um meio para visualizar e acompanhar pelas mudanças das sociedade e do mercado, como determinado conceito acompanhou as evoluções. Com isso, pode-se entender melhor os conceitos e áreas de conhecimento, e aplicar isso no desenvolvimento de novos produtos ou serviços.

Embora encontre-se literaturas que abordem separadamente sobre a história do Design de Serviços e sobre a evolução do conceito "Serviços", percebe-se carência de material que relacione estes dois termos por uma análise histórica. Com isso, espera-se visualizar as necessidades das empresas que originaram essa junção e poder desenvolver melhor os serviços, dependendo do momento do mercado e da sociedade.

O principal objetivo desta pesquisa é relacionar os termos "Serviços" e "Design de Serviços" por meio de uma análise histórica, e visualizar como esses conceitos passaram a se relacionar, integrar e melhorar os processos de desenvolvimento de serviços.

\section{Procedimentos metodológicos}

Inicialmente realizou-se uma breve revisão bibliográfica sobre os assuntos "Serviços" e "Design de Serviços". Por essa pesquisa, percebeu-se publicações em variadas fontes, o que resultou na opção de uma revisão sistemática.

Para a revisão sistemática, primeiramente foram definidas as bases de dados que seriam pesquisadas, sendo escolhidas: Scopus, Wiley, Engineering Village e Emerald. Dentro dessas bases de dados foram usadas três estratégias de palavras-chave para localizar as principais publicações. As publicações encontradas por essas estratégias foram exportadas para um software de gerenciamento bibliográfico, onde foram retirados os artigos duplicados e feita a separação dos arquivos de acordo com o ano, fonte de publicação, autores, e periódicos. Na sequencia, foi realizada uma filtragem para seleção dos artigos que estavam dentro da área de estudo, para enfim analisar as tendências das produções científicas.

Após a revisão sistemática foi feita a leitura dos materiais mais relevantes e a seleção dos dados de maior destaque, e em sequencia construídas linhas do tempo dos termos "Serviços" e "Design de Serviços". Com as linhas do tempo foi realizada uma análise de como ocorreu a convergência entre as duas áreas e refletido sobre 
a importância do Design de Serviços dentro dos processos de desenvolvimento de serviços nos dias atuais.

Na imagem a seguir, Figura 1, é possível visualizar resumidamente a sequencia de procedimentos metodológicos utilizada neste estudo:

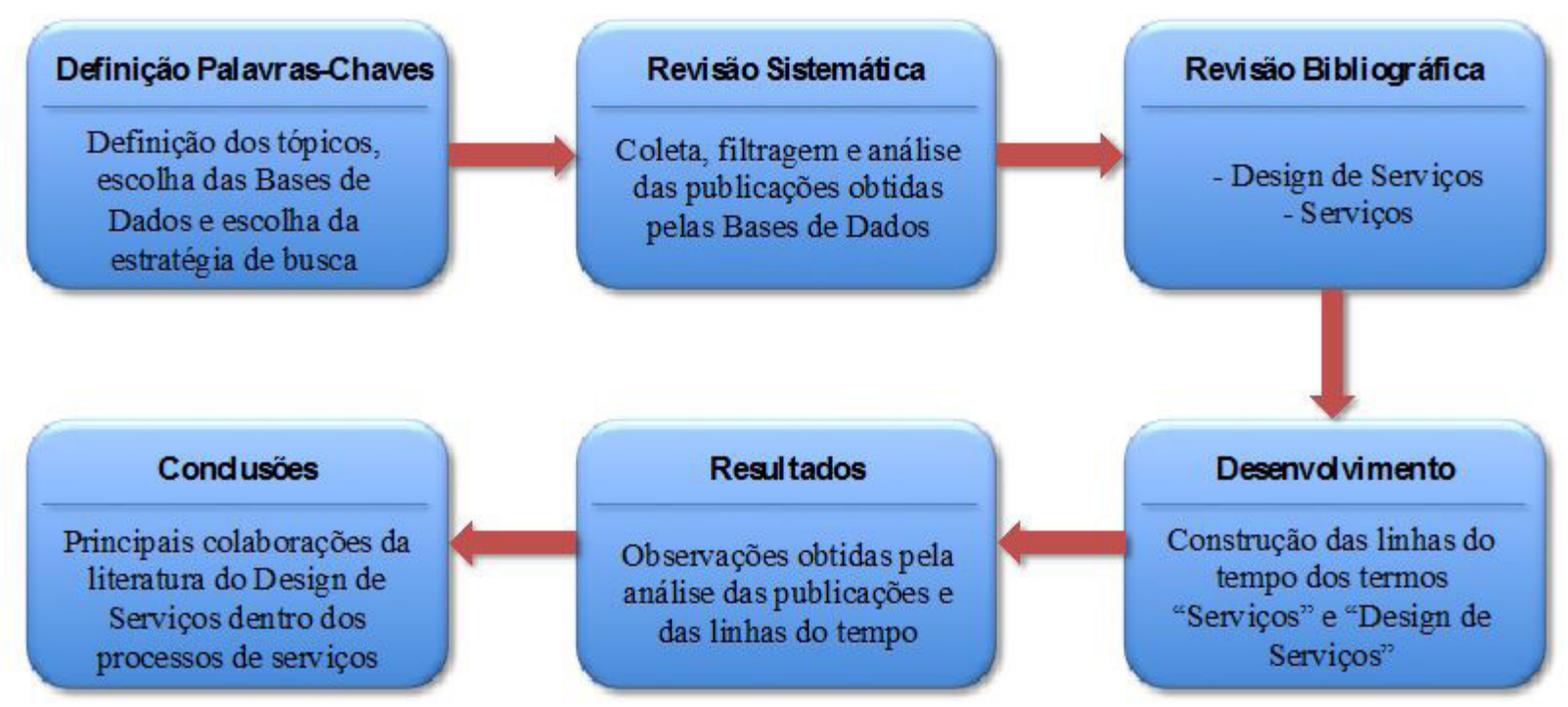

Figura 1 - Sequência dos procedimentos metodológicos. Fonte: Autor.

\subsection{Revisão Sistemática}

Revisão sistemática é uma forma de revisão da literatura que apresenta de maneira clara e explícita um resumo dos principais estudos de determinada área, e mostra os aspectos mais relevantes de forma ampla, em vez de limitar-se à leitura de uma quantidade pequena de trabalhos. A revisão sistemática objetiva nortear o desenvolvimento de um trabalho, pois ela pode indicar novos rumos para próximos estudos, e identificar os principais métodos utilizados na pesquisa em determinada área (SAMPAIO; MANCINI, 2007).

Para a revisão sistemática foram escolhidas quatro bases de dados online: Scopus, Wiley, Engineering Village e Emerald. Delimitou-se a pesquisa para apenas trabalhos de congressos e periódicos.

Inicialmente escolheram-se três termos de buscas para o assunto design: "service design", "design experience" e "design thinking". Para o termo serviços, considerou-se quatro: "service development", "service quality", "service improved" e "PSS" (Product-Service System). Essa seleção foi denominada estratégia 1.

Com os dados obtidos, descartaram-se os trabalhos relacionados à área de $\mathrm{Ci}$ ências da Computação, pois, considerou-se que afastavam-se demais dos assuntos desta pesquisa. Foi aplicada então a estratégia 2, que além da estratégia 1 foram excluídos os trabalhos que contivessem os termos "web" e "net".

Com estes dados, restringiu-se os trabalhos para arquivos apenas em inglês e português (estratégia 3). Os resultados desta busca seguem resumidamente apresentados no Quadro 1: 


\begin{tabular}{cccc}
\hline $\begin{array}{c}\text { Estratégia 1: } \\
\text { Base de } \\
\text { Dados }\end{array}$ & $\begin{array}{c}\text { ("design service" OR "design } \\
\text { thinking" OR "design experience") } \\
\text { AND ("service development" OR } \\
\text { "service quality" OR "service } \\
\text { improved" OR PSS) }\end{array}$ & $\begin{array}{c}\text { Estratégia 2: } \\
\text { Estratégia 1 - } \\
\text { AND NOT } \\
\left(\text { web }^{*} \text { OR net*) }\right.\end{array}$ & $\begin{array}{c}\text { Estratégia 3: } \\
\text { Publicações } \\
\text { em inglês e } \\
\text { português }\end{array}$ \\
\hline $\begin{array}{c}\text { Scopus } \\
\text { Wiley }\end{array}$ & 78 & 56 & 53 \\
$\begin{array}{c}\text { Engineering } \\
\text { Village }\end{array}$ & 711 & 90 & 90 \\
Emerald & 157 & 102 & 100 \\
\hline & 403 & 138 & 138 \\
\hline
\end{tabular}

Quadro 1 - Resultados da revisão sistemática para a etapa de coleta nas bases de dados. Fonte: Autor.

Os 379 resultados obtidos foram exportados para o software Endnote ${ }^{\circledR}{ }^{5}$, onde foram retirados os trabalhos duplicados. Com isso, chegou a um número de 337 inéditos, entre os quais foram selecionados os trabalhos que tinham maior relação com os assuntos da pesquisa. Essa filtragem foi realizada pela leitura dos títulos, palavraschave e resumos, chegando-se ao valor de 247 trabalhos (Figura 2).

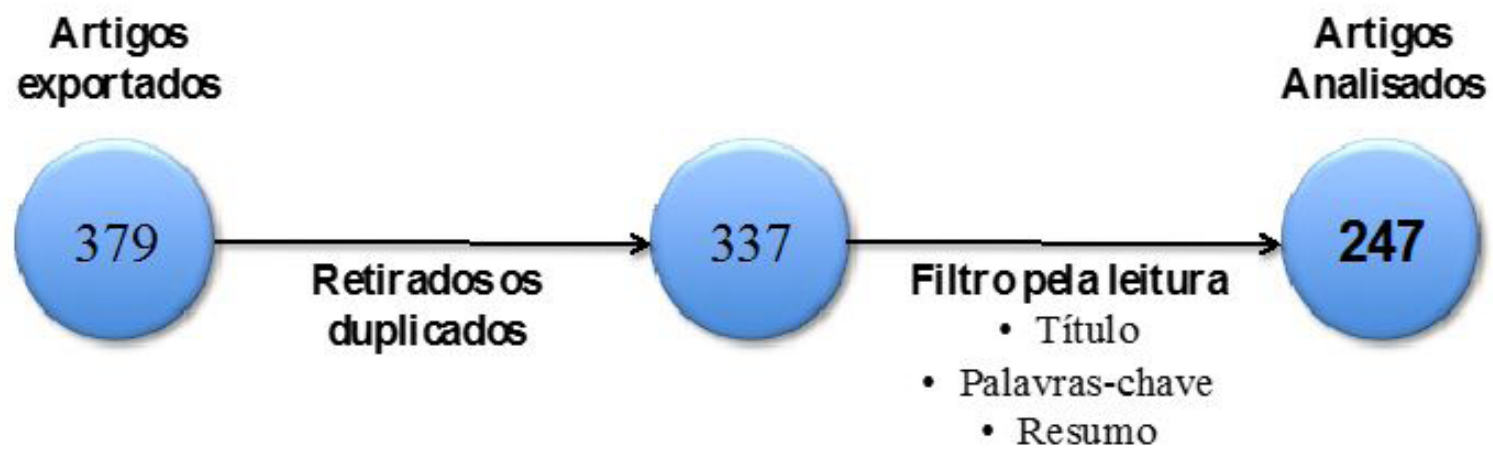

Figura 2 - Método utilizado para filtrar os arquivos localizados pelo Quadro 1. Fonte: Autor.

Dentre os 247 trabalhos selecionados, percebeu-se claramente o aumento das publicações a partir do ano de 1998, com destaque para o crescimento considerável entre 2011 e 2014, como pode ser observado na Figura 3:

5 EndNote® - gestor de referências bibliográficas produzido pela Thomson Scientific. Permite reunir referências bibliográficas de bases de dados online, importar e agrupar de diferentes formas (ENDNOTE, 2015). 


\section{Publicações x Ano}

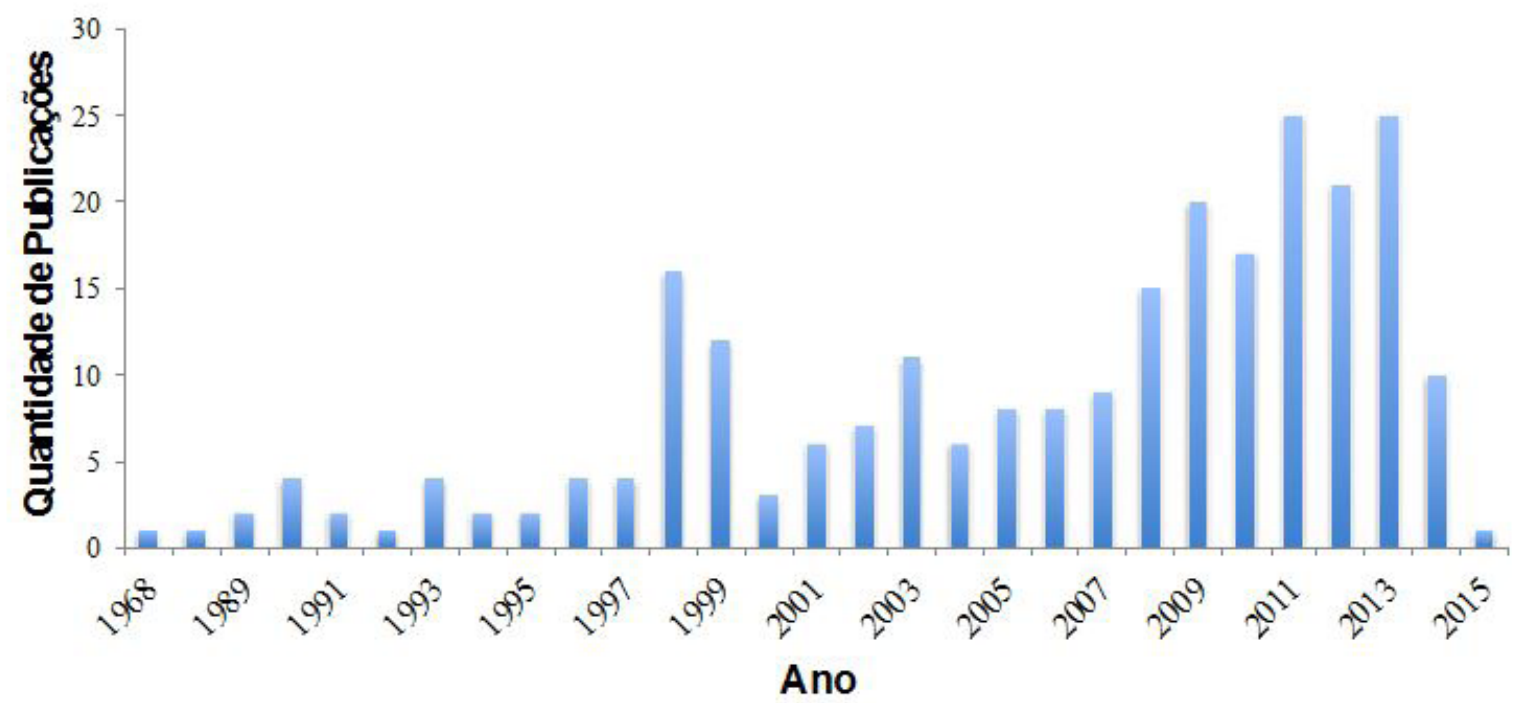

Figura 3 - Relação da quantidade de publicações dentro do grupo selecionado para análise. Fonte: Autor.

Do total de trabalhos selecionados, 60 foram publicados em anais e eventos e 187 em periódicos. Considerando a representatividade dos periódicos, a Figura 4 mostra aqueles que apresentaram mais publicações e seus respectivos fatores de impacto. Na totalidade foram identificados 89 diferentes periódicos, no entanto, para facilitar a visualização, foram colocados no gráfico da Figura 4 apenas os mais citados dentro do grupo de 187 publicações. Utilizou-se como critério aqueles que apresentaram mais de três publicações.

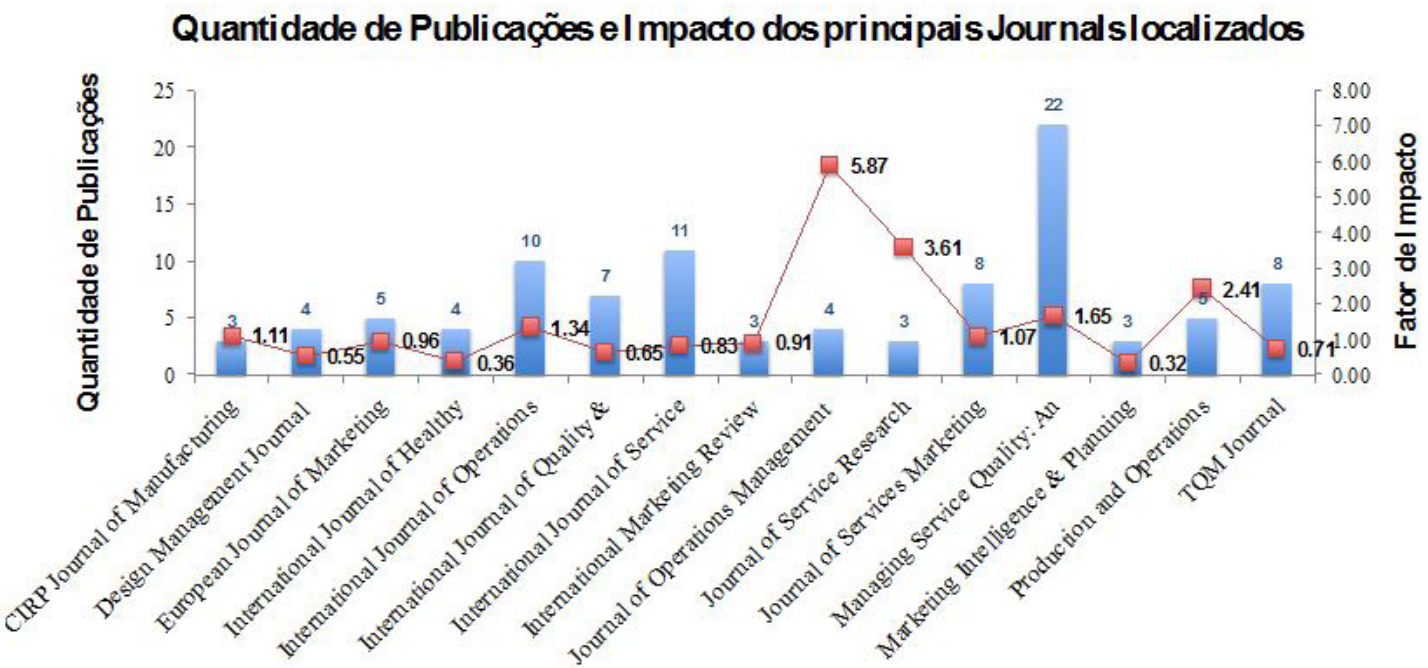

Figura 4 - Relação da quantidade de publicações dos periódicos mais encontrados no grupo resultante do Quadro 1, com os respectivos fatores de impacto. Fonte: Autor.

Os 15 periódicos mostrados no gráfico representam mais de $40 \%$ dos resultados obtidos pela busca (o grupo dos 247). Dentre o grupo apresentado na imagem anterior, percebe-se predominância de periódicos relacionados à serviços, qualidade em serviços e operações.

Significativamente, o periódico que mais apresentou publicações dentro dos 
assuntos pesquisados (total de 22), foi o "Managing Service Quality: an International Journal", um periódico focado na publicação de trabalhos que auxiliem para melhores práticas dentro de empresas de serviços (EMERALD GROUP PUBLISHING, 2015).

\section{Desenvolvimento}

\subsection{Serviços}

Segundo o dicionário Michaelis, 2015, o termo "serviços" pode ser definido como: "Ato ou efeito de servir. Execução de trabalho ou desempenho de funções, ordenados ou pagos por outrem. Ato ou ação útil aos interesses de alguém; bons ofícios; favor, obséquio. Fato de que depende o bem-estar de muitas pessoas."

$\mathrm{Na}$ literatura, diferentes autores conceituam o termo "serviços", mas o que é comum para grande parte das definições é a intangibilidade e o consumo simultâneo, ou seja, é um bem não físico que é utilizado pelos consumidores no momento em que é fabricado (FITZSIMMONS, 2014). Em uma definição mais objetiva, serviços podem ser considerados atividades econômicas que uma parte oferece a outra, e normalmente são baseadas no tempo e no desempenho que oferecem para seus consumidores (LOVELOCK, WIRTZ, HEMZO, 2011).

Setor de Serviços ou setor terciário, é denominado o setor que produz os serviços (bens intangíveis), bem como o destino dos setores primário e secundário (comércio). Exemplos mais comuns de serviços são bancos, restaurantes, serviços educacionais, assistência médica e serviços públicos gerais.

É notável que a porcentagem de participação do setor terciário no cenário econômico está crescendo em grande parte dos os países. Com o desenvolvimento da economia, a participação do emprego nas áreas agrícolas e industrial diminuí, e consequentemente a de serviços aumenta (LOVELOCK, WIRTZ, HEMZO, 2011). Uma das explicações para esta relação de serviços com a economia é que o impulso para o avanço dos serviços foi muito influenciado pelos avanços na tecnologia da informação, pela inovação e pelas expansões geográficas, as quais geraram novas demandas para o mercado.

Considera-se que a história dos serviços iniciou com os Fisiocratas em 1750, que propuseram uma diferenciação entre as atividades agrícolas e não agrícolas (JOSHI, 2008). Embora ainda sem definição precisa, Adam Smith em 1776 propôs a ideia de trabalhos tangíveis (produtos) e trabalhos não tangíveis. No entanto, a primeira tentativa de definição do conceito "serviço" surgiu apenas na década de 1930 com Fischer (1933), que dividiu as atividades econômicas em setores e usou o termo "terciário" para todas as atividades que não fossem agrícolas, nem industriais. No entanto, somente em 1950, Clark sugeriu o conceito "serviço" pela primeira vez (SAMBATTI, RISSATO, 2003).

Após o fim da Segunda Guerra (1945), ocorreu um grande desenvolvimento industrial, período em que os trabalhos e pesquisas relacionados à serviços foram escassos. No entanto, na década de 1960 com a revolução da microeletrônica e globalização das atividades econômicas, ocorreu grande impacto nas áreas de seguros, telecomunicações, transportes e serviços financeiros. 
Em 1973, Daniel Bell afirmou que em pouco tempo o setor terciário seria a principal atividade econômica dos países desenvolvidos. Em 1978, Browning e Silgelmann publicaram um trabalho que apresentaram mais claramente o distanciamento dos serviços da atividade industrial, além de proporem uma classificação para os diferentes serviços.

Na década de 1980, com a ascensão da informatização, ocorreram significativas melhoras na infraestrutura dos serviços (ROGGERO, 1998).

Já na década de 1990 com o processo de concentração e centralização do capital ocorreu uma reestruturação na forma de controle das empresas e na sua administração, o que demandou um aumento da oferta de serviços que auxiliassem as empresas nessa nova forma de organização, como de natureza contábil e financeira, assessoria jurídica, entre outras.

É também no início dos anos de 1990 que surgiu o termo PSS (System ProductService), uma abordagem de negócio em que produtos (físicos) e serviços (imateriais) são unidos com o objetivo de além de atender as necessidades, entregar um valor diferenciado aos clientes (MAUSSANG et al., 2007).

A partir dos anos 2000 ocorreu elevada demanda de serviços, ocasionada principalmente pela urbanização de países em desenvolvimento, pela expansão de programas governamentais e pelos avanços tecnológicos. Na Figura 5 é possível observar resumidamente os principais eventos e trabalhos publicados com relação à serviços ao longo dos tempos.

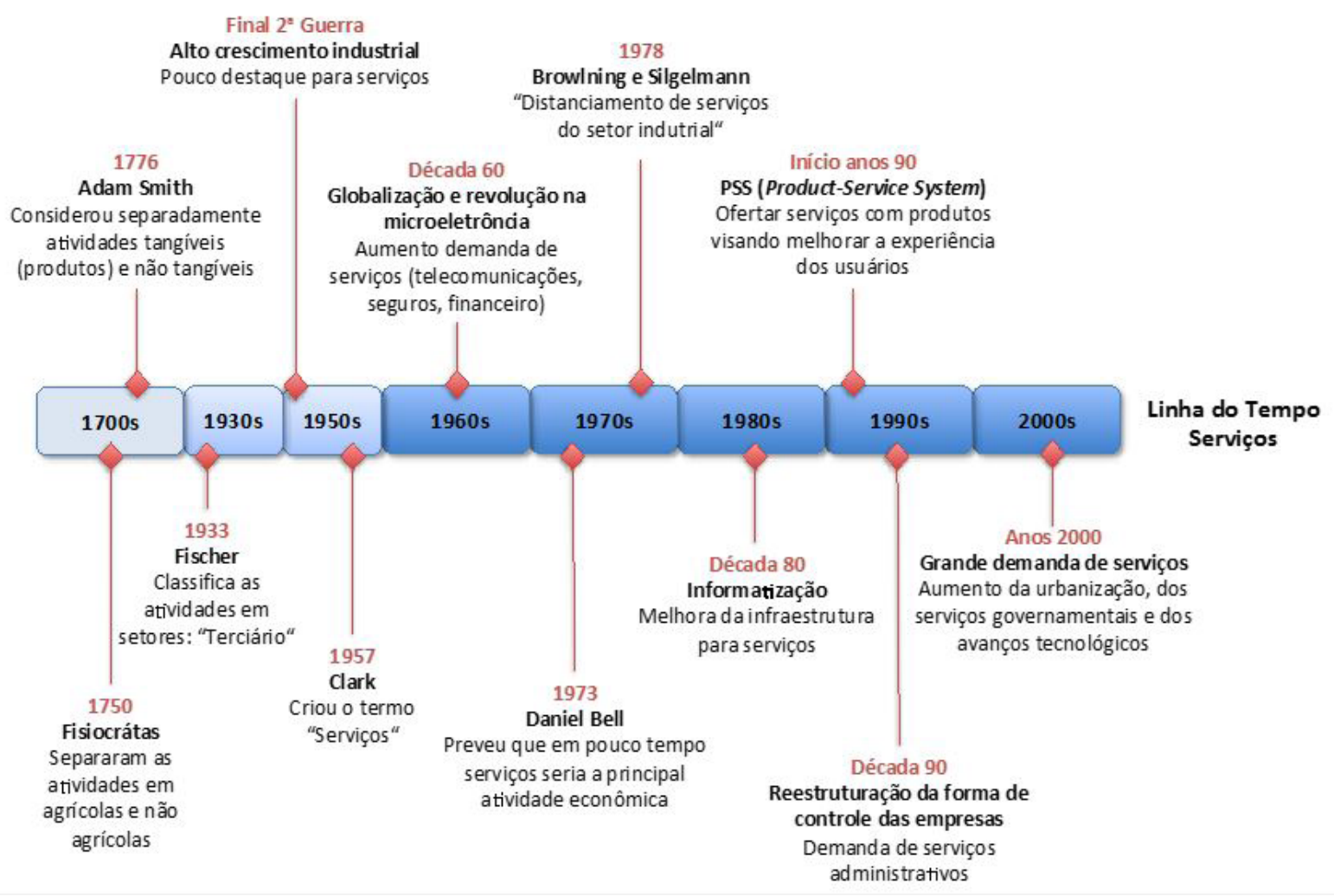

Figura 5 - Linha do Tempo: Serviços. Fonte: Autor.

\subsection{Design de Serviços}


Service Design ou Design de Serviços pode ser considerado como um esforço para desenvolver e fornecer serviços com o máximo de impacto e a melhor experiência possível, tantos para os usuários, como para a organização fornecedora do serviço. Integra as áreas de administração, marketing, pesquisa e design (MORITZ, 2005).

Segundo a literatura (COSTA JUNIOR, 2012), há várias divisões e tarefas propostas por diferentes autores para executar o Design de Serviços. Moritz, 2005, propõe uma divisão simplificada do processo em seis fases distintas e sequenciais:

1. Entendimento - Pesquisar e aprender sobre os clientes, o contexto e o mercado. É a fase de compreender a conexão entre o projeto e a realidade;

2. Pensamento - Fase em que se decide sobre as direções estratégicas do projeto. Identifica-se critérios para desenvolver as estratégias;

3. Geração - Fase que desenvolvem-se os conceitos. Etapa que são criadas ideias novas e criativas;

4. Filtro - Fase que seleciona as melhores ideias. Essa seleção ocorre baseada na combinação de conceitos e avaliação de resultados e soluções;

5. Explicação - Fase que possibilita o entendimento das ideias selecionadas. Isso pode ser feito pela visualização e mapeamento dos processos, ilustrando cenários em potencial e apresentando as futuras possibilidades;

6. Realização - Momento em que faz-se acontecer. Desenvolve-se e aplica-se soluções, protótipos e processos. A importância dessa fase deve-se ao fato de assegurar a melhor performance do serviço (MORITZ, 2005).

O Design de Serviços surgiu quando percebeu-se que alguns serviços apresentavam muitos problemas relacionados à qualidade, e como o design era utilizado para auxiliar no desenvolvimento de produtos, passou-se a utilizar a mesma ideia de aplicação para serviços (MORITZ, 2005).

Umas das primeiras pesquisas envolvendo Design de Serviços ocorreu em 1984, quando Lynn Shostack publicou um artigo explicando como projetar um serviço. 0 mesmo autor lançou em 1984 o trabalho "Design Services that deliver", que introduziu a ferramenta blueprinting (MELLO et al., 2010).

Blueprinting é um mapa com representação de todas as transações que constituem a entrega de um serviço por uma empresa. No princípio, era utilizado para mostrar como deveria ser um produto, bem como as especificações necessárias para a manufatura. Lynn Shostack afirmou que os serviços também poderiam ser representados por um diagrama visual, e desta forma propôs um blueprinting para serviços (FITZSIMMONS, 2014).

Em 1991 é lançado o livro "Total Design" por Gill e Bill Hollings, que incluíram ao Design de Serviços a perspectiva da administração, e afirmaram que os serviços eram como produtos e que deveriam ser projetados pela Gestão do Design (COSTA JUNIOR, 2012). No mesmo ano, a Universidade de Ciências Aplicadas de Colônia - KISD (Köln International School of Design), na Alemanha, torna-se a primeira universidade do mundo a oferecer educação em Service Design, o que foi idealizado por Michael Erlhoff e Birgit Mager.

Em 1993, Joseph Pine lança o trabalho "Mass Costumization: The new frontier in Business Competition", que apresenta as mudanças que as empresas estavam vivendo, passando de ofertas padronizadas para a produção de ofertas customizadas de 
bens, serviços e experiências (COSTA JUNIOR, 2012).

Em 1995 Birgit Mager foi considerada a primeira professora da área de Service Design, o que contribuiu para considerável aumento no número de publicações na área (MORITZ, 2005).

Em 2001 a Live/Work, de Londres lançou a primeira consultoria em Service Design, e em 2002 a IDEO (A Design and Innovation Consulting Firm - Consultoria Internacional de Design) também passou a oferecer esse serviço.

Em 2004 Birgit Mager estabeleceu o Service Design Network, uma instituição especialista em Service Design, com o objetivo de divulgar e disseminar essa área de conhecimento. Até os dias atuais essa instituição promove congressos e eventos para divulgação e integração de trabalhos (SDN, 2015).

Segundo Costa Junior, 2012, foi somente em 2005 que as pesquisas envolvendo Design de Serviços passaram a ganhar destaque no Brasil. Uma visualização resumida dos principais eventos e trabalhos acerca do tema pode ser visualizada na Figura 6 :

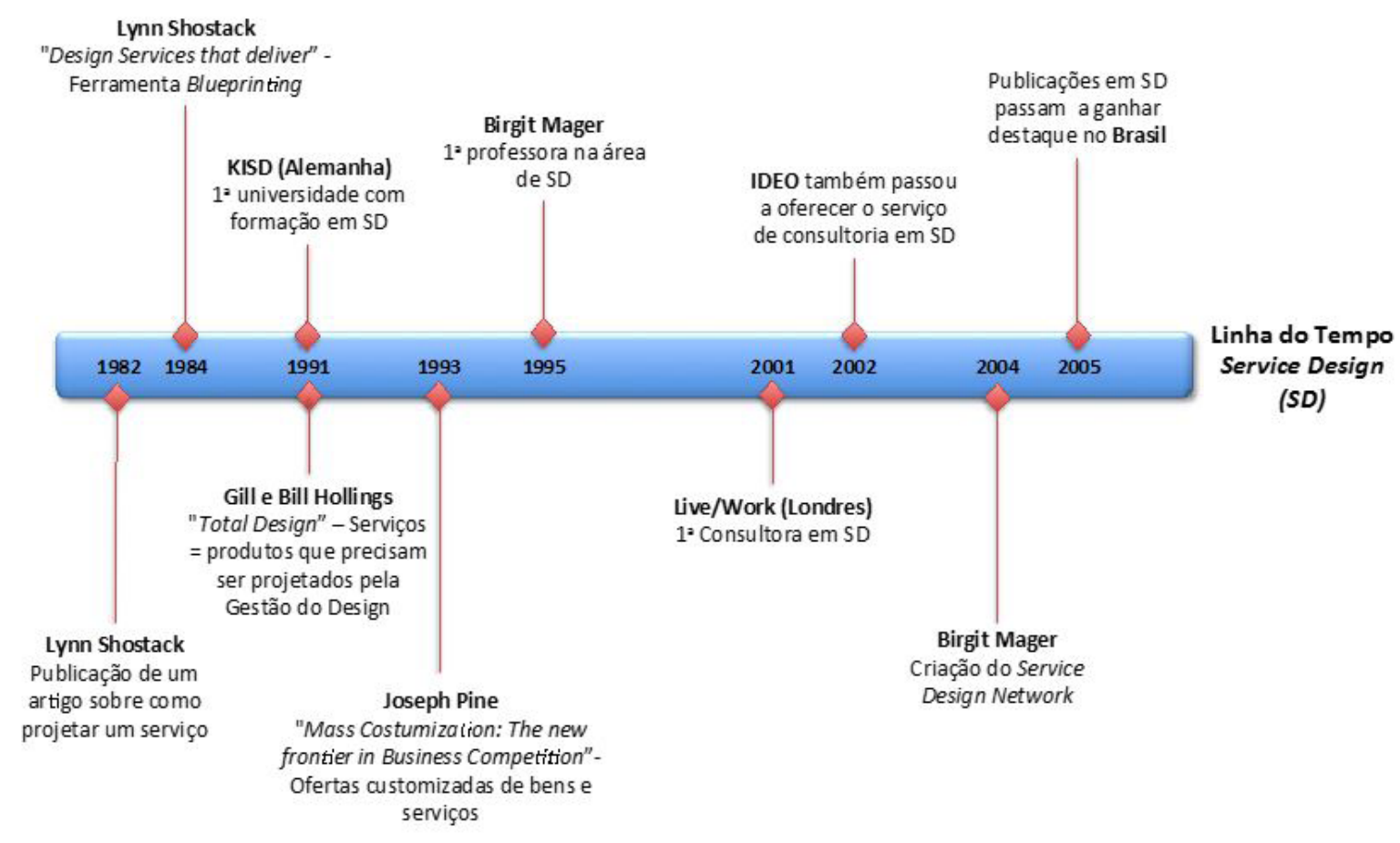

Figura 6 - Linha do Tempo: Design de Serviços. Fonte: Autor.

Como pode-se visualizar na linha do tempo, e de acordo com o que afirma Moritz, 2005, o Design de Serviços é um campo de conhecimento relativamente novo, tendo maior destaque nos últimos vinte anos, mostrando para as empresas uma nova perspectiva de inovação, melhorias e comunicação pelas organizações para com os consumidores.

\subsection{Resultados e Discussões}

Considerando as linhas do tempo apresentadas nas Figura 5 e Figura 6, foram selecionados os principais eventos e publicações ocorridos concomitante entre Serviços e Design de Serviços, e apresentados em uma nova linha do tempo, conforme 
pode ser visualizado na Figura 7:

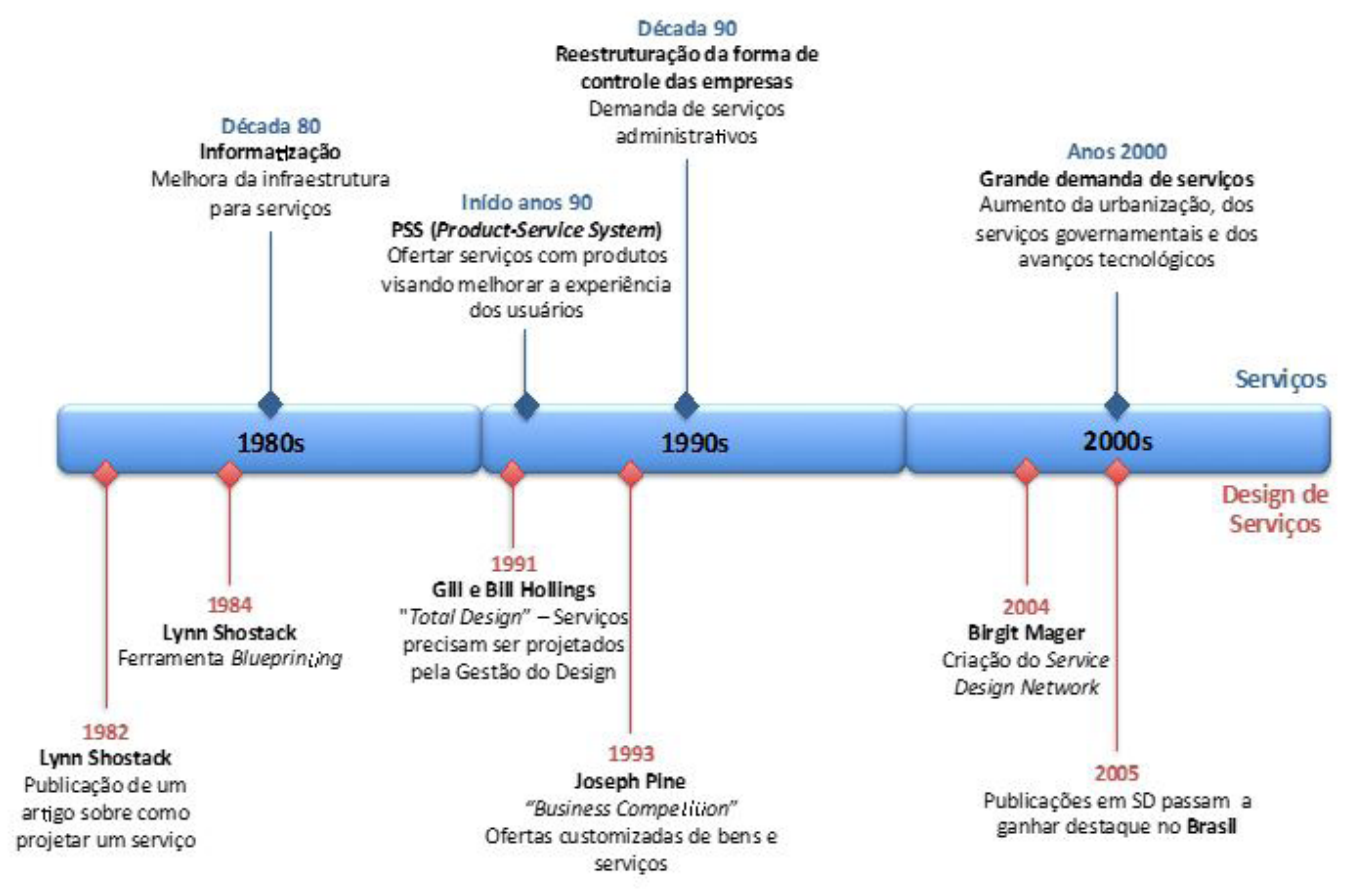

Figura 7 - Convergência entre as linhas do tempo de "Serviços" e "Design de Serviços". Fonte: Autor.

Como é possível observar, o Design de Serviços passou a destacar-se a partir da década de 1980, momento em que o serviços modernizaram-se e passaram a utilizar novas ferramentas obtidas principalmente pela informatização.

Observa-se que o Design de Serviços passou a estar presente dentro do contexto de serviços a partir do significativo aumento da oferta de serviços no setor econômico. Esse fato mostra-se em concordância com o que afirma Joseph Pine, 2011, sobre o progresso do valor econômico. Segundo o autor, os produtos já passaram e continuam evoluindo ao longo do tempo por quatro fases distintas e sequenciais: commodities (matéria-prima), bens, serviços e experiências (PINE, 2011). E nessa evolução, há uma diferenciação que progride proporcionalmente com as necessidades dos clientes.

Com essa nova exigência de proporcionar experiências aos usuários, emergiu a necessidade de analisar e projetar melhor os serviços, tarefa que culminou no Design de Serviços. Dentro desse contexto, destaca-se a Gestão de Design dentro de empresas, que como afirma Mozota, 2002, se fundamenta em identificar e comunicar as maneiras pelas quais o design pode contribuir para as estratégias das empresas, ou seja, entende-se como a forma que o design está presente dentro das organizações para visualizar e projetar produtos e serviços de acordo com as necessidades das sociedades.

O aumento da demanda de serviços na década de 1990 colaborou também para grande crescimento na produção científica (Figura 3). Destaque para alguns autores na área de Design de Serviços, como Lynn Shotaske, que publicou os primeiros trabalhos que envolviam projetos de serviços. Birgit Mager também teve e continua tendo grande destaque na área, com atuação principalmente pelo Service Design Network que contribui com frequentes eventos e publicações. 
Dentre os resultados obtidos pela revisão sistemática, notou-se que entre os periódicos que mais apresentaram publicações há predominância daqueles voltados para a qualidade de serviços e melhores práticas em empresas de serviços. Esses dados mostram a tendência de estudos focados na qualidade dos serviços, fato possivelmente apoiado pelas crescentes exigências dos consumidores, que com a ampliação de ofertas, culminou em novas necessidades.

O Design de Serviços passou a destacar-se dentro dos processos de desenvolvimento de serviços a partir do momento que as pessoas adquiriram mais conhecimentos e consequentemente aumentaram níveis de exigência, passando a exigir do governo e das empresas privadas serviços diferenciados e com maior qualidade, visando novas e melhores experiências. Nota-se que da mesma forma que o design procurava resolver problemas dentro do contexto de desenvolvimento de produtos, o mesmo destacou-se como uma opção para projetar os serviços, ofertando assim novas experiências aos usuários, e isso foi possível com a atuação da Gestão do Design dentro das organizações.

\section{CONCLUSÕES}

Como se observou na revisão histórica, o setor de serviços está presente na sociedade há bastante tempo, no entanto, devido às mudanças ocorridas principalmente pelo progresso econômico, sofreu significativas modificações ao longo do tempo. O Design de Serviços, iniciado na década de 1980, surgiu como uma opção de entender os contextos ambientais e temporais e projetar melhor os serviços, e com isso ofertar novas e melhores experiências aos usuários, apoiado pela Gestão de Design dentro das empresas.

A revisão sistemática dos termos em estudo comprovou a tendência de aumento de pesquisas relacionadas à qualidade de serviços, envolvendo periódicos de alto impacto na pesquisa acadêmica desde a década de 1990.

Essa tendência mostrada por publicações, reflete a importância da produção científica nessa área de estudo, pois, como visto há uma grande relação deste aumento e melhora de serviços com a inovação e o desenvolvimento econômico dos países.

\section{Referências}

COSTA JUNIOR, Jairo da. Roposição de um modelo de referência para o design de serviços ecoeficientes em sistemas produto-serviço. 2012. 201 f. Dissertação (Mestrado) - Curso de Design, Universidade Federal do Paraná, Curitiba, 2012.

DZHAIN, Nikita. Development of the Tertiary Sector: A Comparative Analysis Between BRIC Countries and Select Developed Economies. 2012. 54 f. Monografia (Especialização) - Curso de International Business And Logistics, Helsinki Metropolia University Of Applied Sciences, Vanda, 2012.

EMERALD GROUP PUBLISHING - Journal: Managing Service Quality: an International 
Journal - Disponível em: http://www.emeraldgrouppublishing.com/products/journals/journals.htm?id=jstp. Acesso em: 22 junho de 2015.

Endnote for Windows: Bibliographies Made Easy. Version X5. Thomson Reuters, 2015. 1 CD-ROM

FITZSIMMONS, James A.; FITZSIMMONS, Mona J. Administração de serviços: operações, estratégia e tecnologia de informação. 7a ed. Porto Alegre: Bookman, 2014.

IBGE - Instituto Brasileiro de Geografia e Estatística - Disponível em: http://www. ibge.gov.br/home/. Acesso em: 26 abril de 2015.

JOSHI, Seema. Growth and Structure of Tertiary Sector in Developing Economies. Nova Deli: Independent Publishe, 2008. 244 p.

LOVELOCK, Christopher; WIRTZ, Jochen; HEMZO, Miguel Angelo. Marketing de Serviços: Pessoas, Tecnologia e Estratégia. 7. ed. São Paulo: Pearson Prentice Hall, 2011. $528 \mathrm{p}$.

MAUSSANG, Nicolas et al. A model for designing product-service systems using functional analysis and agent based model. In: INTERNATIONAL CONFERENCE ON ENGINEERING DESIGN, ICED'07, 2007, Paris. Conferência. Paris, 2007. p. 809 - 820.

Mello., and Carlos Henrique Pereira et al.. Gestão do processo de desenvolvimento de serviços. Atlas, 2010. VitalBook file.

MELO, H. et al. O setor de serviços no Brasil: uma visão global - 1985/95. Rio de Janeiro: IPEA, 1998.

MICHAELIS. Dicionário de Português Online: Moderno Dicionário da Língua Portuguesa. Disponível em: <http://michaelis.uol.com.br/moderno/portugues/index. php>. Acesso em: 10 jun. 2015.

MORITZ, S. M.; (2005) Service Design - Practical Access to an Evolving Field (Dissertação de Mestrado: Köln International School of Design) Acessado em 13 de abril de 2015, em: http://stefanmoritz.com/_files/Practical\%20Access\%20to\%20Service\%20 Design.pdf

MOZOTA, Brigitte Borja de. Design and competitive edge: A model for design management excellence in European SMEs. Design Management Journal: Academic Review, Boston, v. 2, 2002. Quartely.

PINE, Joseph; GILMORE, James. The Experience Economy: Updated Edition. Boston: Havard Business Publishing, 2011. 362 p. 
ROGGERO, Rosemary. Uma Leitura Sobre o Desenvolvimento do Setor Terciário no Movimento Contemporâneo do Capital. Boletim Técnico do Senac, Rio de Janeiro, v. 24, n. 3, p.2-15, set. 1998. Trimestral.

SAMBATTI, A. P.; RISSATO, D . O setor terciário da economia: uma discussão teórica introdutória. In: II Seminário do Centro de Ciências Sociais Aplicadas de Cascavel Ciências Sociais Aplicadas na era dos serviços, 2003, Cascavel-PR.

SAMPAIO, R C; MANCINI, M C. Estudos de revisão Sistemática: Um guia para síntese criteriosa da evidência científica. Revista Brasileira de Fisioterapia, São Carlos, v. 11, n. 1, p.83-89, jan. 2007. Trimestral.

SDN - Service Design Network - Disponível em: http://www.service-design-network.org. Acesso em 27 abril de 2015. 